\title{
Effects of breathing on neck volume during supine position
}

\author{
Lynn M. Baniak ${ }^{* 1,2}$, Carolyn S. Pierce ${ }^{1,2}$, Ken J. McLeod ${ }^{1,3,4}$ \\ ${ }^{1}$ Clinical Science and Engineering Research Center, Binghamton University, Binghamton, United States \\ ${ }^{2}$ Decker School of Nursing, Binghamton University, Binghamton, United States \\ ${ }^{3}$ Department of Entrepreneurship \& Innovation Partnerships, Binghamton University, Binghamton, United States \\ ${ }^{4}$ Department of Systems Science and Industrial Engineering, Binghamton University, Binghamton, United States
}

Received: June 24, 2015

DOI: $10.5430 / \mathrm{cns} . v 3 \mathrm{n} 4 \mathrm{p} 88$
Accepted: August 24, 2015

URL: http://dx.doi.org/10.5430/cns.v3n4p88

\begin{abstract}
Objective: Fluid which accumulates in the lower extremities during the day may redistribute towards the head (rostral fluid shift) during sleep, and so may play a role in restricting the upper airway leading to sleep disordered breathing (SDB). In this pilot study, six healthy men and women were recruited to test the hypothesis that the transition to a recumbent position results in a significant increase in neck fluid volume.

Methods: Change in neck volume (NV) was measured using air plethysmography during 30 minutes of sitting (Sit) and 45 minutes following transition to a supine position (Supine). Each subject was assessed four times on separate days. Linear regression and spectral analysis were undertaken to characterize NV changes.

Results: Three men and three women (two each in their $20 \mathrm{~s}, 40 \mathrm{~s}$, and $60 \mathrm{~s}$ ) participated. BMI averaged $24.45 \pm 3.9 \mathrm{~kg} / \mathrm{m}^{2}$. Both average Sit and Supine NV shifts were significantly greater than zero, $+4.33 \mathrm{ml} / \mathrm{hr} \pm 1.63 ; 95 \%$ CI $[1.07,7.59] ;+1.51 \mathrm{ml} / \mathrm{hr}$ $\pm 0.55 ; 95 \%$ CI [0.41, 2.61] $(p<.05)$, respectively, however there was no significant difference between Sit and Supine. Spectral analysis of $\mathrm{NV}$ recordings revealed peaks at $\sim 0.25 \mathrm{~Hz}$ and $\sim 1.2 \mathrm{~Hz}$, consistent with respiration and heart contraction rates, respectively. Average respiratory NV shift was $0.03 \mathrm{ml} \pm 0.01 ; 95 \%$ CI [0.01-0.05] (Sitting) and $0.08 \mathrm{ml} \pm 0.02$; $95 \%$ CI [0.04, 0.12 ] (Supine), both significantly greater than zero $(p<.05)$, with supine fluid shifts significantly greater than during sitting $(p<$ $.01)$.

Conclusions: These results suggest that respiratory activity may have the dominant influence on NV when supine, potentially contributing to SDB. Further study of neck fluid shifts during respiration and its influence on sleep quality in persons with SDB is proposed.
\end{abstract}

Key Words: Rostral fluid shift, Air plethysmography, Sleep disordered breathing, Sleep quality

\section{INTRODUCTION}

In the upright position, gravitational force operating on the fluid in the body results in substantial fluid pooling in the lower extremities. This volume includes intravascular fluid shifts (venous pooling) of approximately one liter ${ }^{[1]}$ and a similar amount of interstitial fluid extravasating from the cap- illaries. ${ }^{[2]}$ At the same time, the veins above the heart tend to collapse as blood is returned to the heart by gravity dropping venous pressure to below zero in the upper body. ${ }^{[1,3]}$

When assuming a recumbent position, gravitational forces on the cardiovascular system are much reduced, and venous and interstitial fluid redistributes towards the thorax and head

\footnotetext{
*Correspondence: Lynn M. Baniak; Email: lbaniak1@binghamton.edu; Address: Binghamton University, AB318 Box 6000, Binghamton, NY 13902, United States.
} 
(rostral fluid shift [RFS]). ${ }^{[4-6]}$ This fluid refills low pressure areas including the veins above the heart and has been proposed to increase neck fluid volume resulting in neck tissue expansion and upper airway restriction. ${ }^{[1,7,8]}$ Correspondingly, RFS have been implicated in the development of Sleep Disordered Breathing (SDB) lending to sleep disturbance. ${ }^{[8,9]}$ Additionally, the amount of neck volume (NV) has been positively correlated to neck circumference and the apnea-hypopnea index in men without heart failure. ${ }^{[10]}$

The influence of RFS on the upper airway is not completely understood and testing the hypothesis that supine rest leads to RFS is difficult, as a reliable method for measuring RFS has not been well established. The research on neck expansion following the transition to recumbency has primarily relied on measures of neck circumference, a challenging measure to perform reliably. There is a limited region of the neck not encapsulated by bone and so able to expand externally with neck soft tissue volume changes. While some investigators have utilized simple tape measurements, ${ }^{[9-11]}$ more sophisticated approaches such as liquid metal strain gauge plethysmography (LMSGP) have been utilized. ${ }^{[12,13]}$ Plethysmography is the measurement of volume and volume displacement. In LMSGP, a strain gauge is wrapped around a limb measuring changes in circumference of that limb due to blood flow. While LMSGP allows for dynamic assessment of $\mathrm{NV}$, the tendency of these gauges to roll due to the tapered shape of the neck represents a continuing challenge.

An alternative approach for assessing neck volume change is air plethysmography (APG). ${ }^{[14]}$ Air plethysmography is a noninvasive technique that provides the capability for continuous monitoring of temporal volume dynamics consisting of placing a tubular air chamber around the extremity and connecting the cuff to a pressure transducer and recorder. The air chamber is then inflated with air and any changes in volume of the extremity correspond to changes in the pressure of the air chamber. Thus, these changes are measured in milliliters according to the calibration. ${ }^{[14]}$ We have previously used APG to assess fluid shifts in the lower extremities, yielding consistent and reliable results, contributing to our understanding of venous and interstitial pooling. ${ }^{[15]}$ Because of the sensitivity and reliability of this technology, we investigated RFS within the neck using APG to address the hypothesis that transition to a recumbent position results in a significant increase in neck volume.

\section{Methods}

The study protocol was approved by the Institutional Review Board at Binghamton University, Binghamton, New York. Assessments were performed in the Clinical Science and Engineering Research Center at Binghamton University from
January through April 2014.

\subsection{Participants}

A convenience sample of healthy adult men and women were recruited by word-of-mouth to test the hypothesis that significant RFS occurs when transitioning from a condition of extended quiet sitting to the recumbent position. Inclusion criteria were adult men and women between the ages of 21 and 65 years of age, capable of understanding English, and willing to be tested on four separate occasions for repeat assessments. Exclusion criteria included pregnant women, cognitive impairment, nighttime oxygen use, unable to tolerate prolonged recumbency, history of a deep vein thrombosis or pulmonary embolus, cardiovascular disease, or any abnormality of the neck.

\subsection{APG assessment}

NV change was used as a surrogate measure of neck fluid shifts. A custom APG neck cuff was developed using a Schraeder Valve Tube in which the valve core was removed and ends were sealed (see Figure 1). The cuff was inflated to an operating pressure of $2 \mathrm{mmHg}$. Dynamic pressure output from the air plethysmograph unit was digitized at $10 \mathrm{~Hz}$ following pre-filtering at $10 \mathrm{~Hz}$.

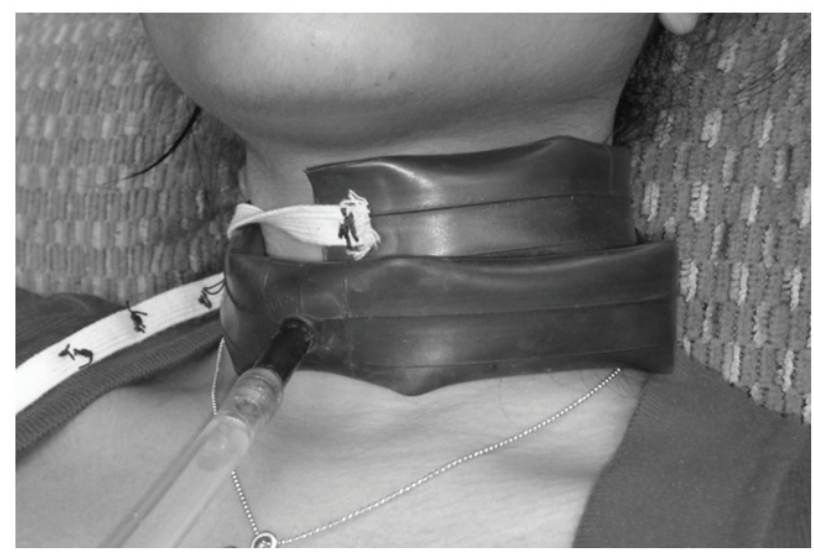

Figure 1. Custom APG neck cuff

\subsection{Protocol}

Subjects were seated in an electrically controlled recliner in an upright position with uncrossed legs. Neck circumference was obtained using a tape measure $(\mathrm{cm})$ and the APG neck cuff was placed around the subject's neck (see Figure 1). Calibration was accomplished using 1 milliliter $(\mathrm{ml})$ boluses of air injected at 1 minute intervals to permit conversion of APG pressures (psi) changes into a standard measure $(\mathrm{ml})$ independent of neck size. The subject's head was positioned to prevent the cuff from touching the back of the chair. 
As shown in Figure 1, the APG cuff used for monitoring neck volume changes was made from a Schraeder Valve Tube with the valve core removed and ends sealed. Elastic bands with multiple hooks were sewn on to adjust for various neck sizes. Once placed in position, the cuff was inflated to a bias pressure of $2 \mathrm{mmHg}$ and calibrated to permit estimation of neck volume changes.

Neck APG data was collected during 30 minutes of quiet sitting (Sit), then during 45 minutes immediately after the subject transitioned to a recumbent position (Supine). Subjects returned for three additional testing sessions utilizing the same protocol ( 6 subjects $\times 4$ assessments on separate days).

\subsection{Data analysis}

Raw APG data as noted in Figure 2 demonstrated an overall trend in neck volume over time, as well as respiratory and cardiovascular effects on neck volume. Each $\sim 3.5$ second peak corresponds to each breathe, and each $\sim 0.8$ second peak corresponds to each heart contraction.

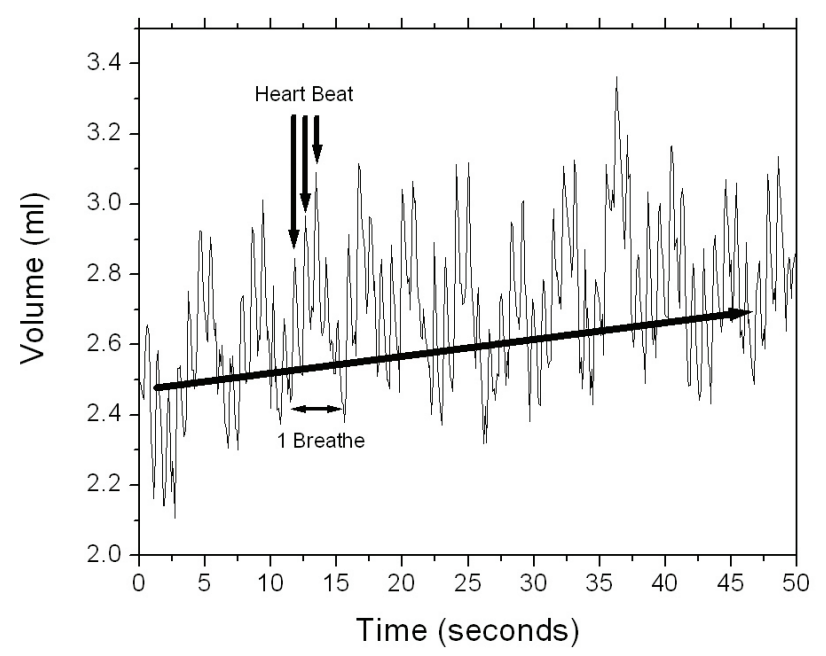

Figure 2. A sample of raw APG data

Linear regression was used to evaluate the trend in neck volume over time and spectral analysis (Fourier Analysis) was then utilized to identify the dynamic respiratory and cardiovascular changes in NV when seated and supine. Spectral analysis converts the input signal from the temporal domain to the frequency domain. Identified peaks in the frequency domain are indicative of significant energy at specific frequencies (repetition rates). Single population T-tests were used to evaluate whether the NV changes during Sit and Supine were significant, and paired T-tests were used to evaluate if the two exposure conditions produced different levels of fluid shift. Analyses were performed using Origin, version
6.1. All data are expressed as means $\pm \mathrm{SE} ; p$ values $<.05$ were considered significant.

\section{RESULTS}

A convenience sample of six healthy subjects without sleep complaints was tested. This sample included three men and three women, aged 20-65 years, two each from their $20 \mathrm{~s}$, $40 \mathrm{~s}$, and $60 \mathrm{~s}$. Initial neck circumference averaged $35.4 \mathrm{~cm}$ \pm 2.89 , and BMI averaged $24.45 \mathrm{~kg} / \mathrm{m}^{2} \pm 3.9$.

Thirty minutes of quiet sitting was associated with average NV changes of $+4.33 \mathrm{ml} / \mathrm{hr} \pm 1.63 ; 95 \% \mathrm{CI}[1.07,7.59]$ (see Figure 3$)$, significantly greater than zero $(p<.05)$. During 45 minutes in the supine position, average $\mathrm{NV}$ changes were $+1.51 \mathrm{ml} / \mathrm{hr} \pm 0.55 ; 95 \% \mathrm{CI}[0.41,2.61]$ also significantly greater than zero $(p<.05)$. Though the supine NV change was less than one-half than observed during sitting, this difference was not found to be significant.

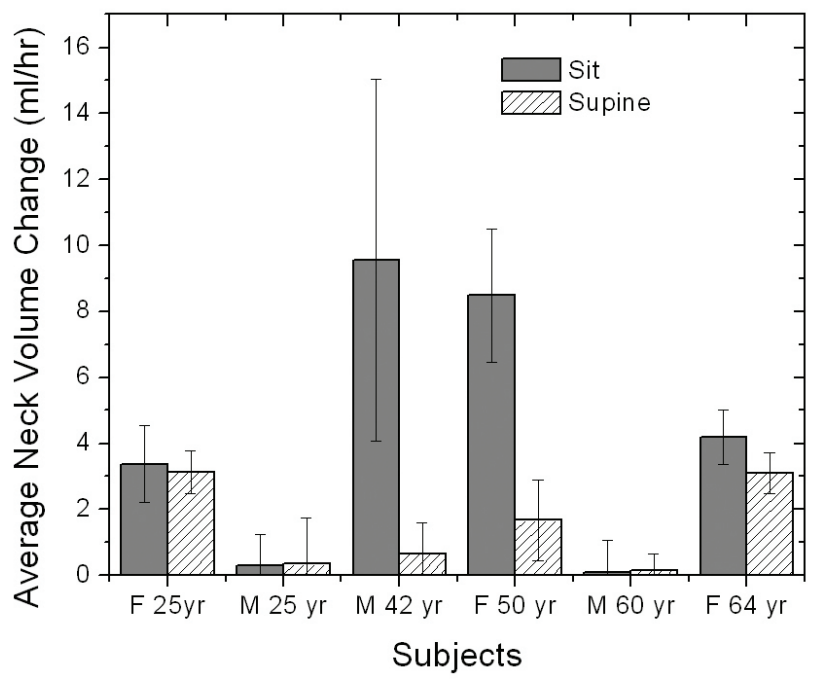

Figure 3. Effect of repositioning on neck volume Neck volume change for each subject was averaged over four testing sessions. Linear regression revealed an average neck volume shift during 30 minutes of sitting of $+4.33 \mathrm{ml} / \mathrm{hr} \pm 1.63$; 95\% CI [1.07, 7.59] and $+1.51 \mathrm{ml} / \mathrm{hr} \pm 0.55$; 95\% CI [0.41, 2.61] in the 45 minutes immediately after transition to the supine position, both significantly greater than zero $(p<.05)$. Though the supine fluid shift was less than one-half than observed during sitting, this difference was not significant.

Spectral analysis of the APG recordings consistently revealed peaks at $\sim 0.25 \mathrm{~Hz}$ consistent with a neck volume change occurring with respirations, and $\sim 1.2 \mathrm{~Hz}$ consistent with a neck volume change associated with heart contractions (see Figure 4).

Respiratory neck volume change for each subject was averaged over four testing sessions for quiet sitting and supine 
(see Table 1).

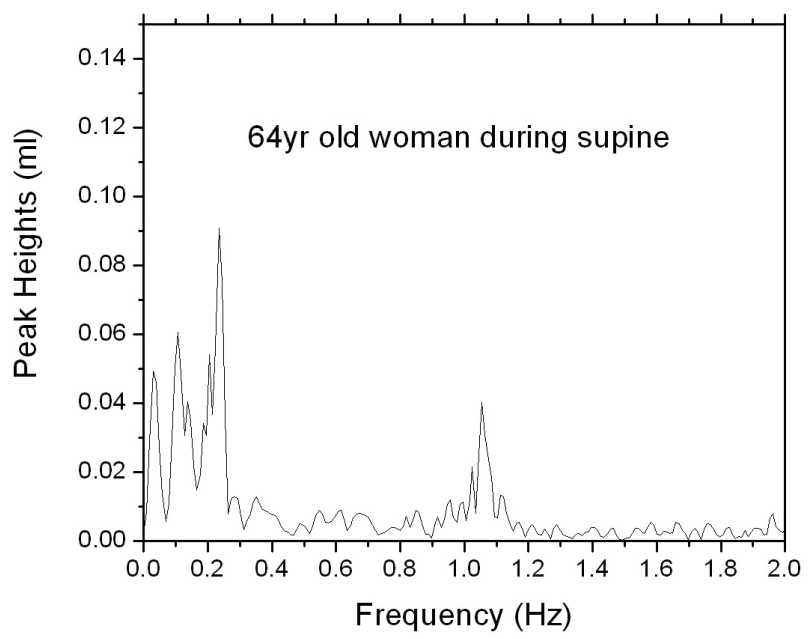

Figure 4. Typical spectral analysis of air plethysmography data

Typical spectral analysis of air plethysmography data from a one minute recording interval while supine. Two distinct peaks, a low frequency peak at $\sim 0.23 \mathrm{~Hz}$ corresponding to respiration, and a second peak at $\sim 1.1 \mathrm{~Hz}$, associated with heart contraction, were evident. Peak heights represent degree of neck fluid volume shifts.

Figure 5 shows the average respiratory NV change during the four testing sessions among all subjects. Spectral analysis demonstrated a greater neck volume change during inhalation when supine in all subjects compared to sitting. Overall Supine $(0.08 \mathrm{ml} \pm 0.02 \mathrm{ml} ; 95 \% \mathrm{CI}[0.04,0.12])$ was significantly greater than overall Sit $(0.03 \mathrm{ml} \pm 0.01 \mathrm{ml} ; 95 \% \mathrm{CI}$ $[0.01,0.05])(p<.01)$. Average heart contraction NV change during sitting was $0.06 \mathrm{ml} \pm 0.008 \mathrm{ml} ; 95 \% \mathrm{CI}$ [0.044, 0.068] and during Supine was $0.06 \mathrm{ml} \pm 0.005 \mathrm{ml}$; 95\%CI [0.05; 0.07], which was not significantly different.

Table 1. Average respiratory NV change during quiet sitting and supine for each subject

\begin{tabular}{lll}
\hline Subject & Quiet Sitting (ml) & Supine (ml) \\
\hline F25 & $0.013 \pm 0.005$ & $0.016 \pm 0.001$ \\
M25 & $0.010 \pm 0.002$ & $0.048 \pm 0.009$ \\
M42 & $0.066 \pm 0.007$ & $0.142 \pm 0.056$ \\
F50 & $0.023 \pm 0.008$ & $0.049 \pm 0.009$ \\
M60 & $0.057 \pm 0.008$ & $0.121 \pm 0.016$ \\
F65 & $0.025 \pm 0.006$ & $0.073 \pm 0.033$ \\
\hline
\end{tabular}

Note. $\mathrm{ml}=$ milliliters; $\mathrm{F}=$ female; $\mathrm{M}=$ male. Values are expressed as mean \pm standard error

\section{Discussion}

The primary goal of this study was to investigate the dynamics in NV using APG to address the hypothesis that transition from a sitting to supine position results in a significant increase in NV. It has previously been suggested that repositioning to the supine position results in increased fluid shift into the neck lending to SDB. ${ }^{[4,7,16]}$ Our results obtained from 20-65 year old subjects using neck APG assessment have confirmed that $\mathrm{NV}$ does increase during recumbency, however, a similar, and possibly higher rate of increase was also observed during quiet sitting. This could occur due to the fact that sitting raises intra-abdominal pressure, increasing the pressure gradient between the abdomen and the thorax, causing abdominal blood to flow towards the thorax and head, resulting in increased fluid in the neck and therefore neck volume. ${ }^{[1]}$ In individuals with SDB, symptoms are substantially greater during sleep in the supine position as compared to when the head is elevated. ${ }^{[17]}$ Since an insignificant difference in $\mathrm{NV}$ change was observed during supine as compared to sitting, we would suggest that an alternative factor may be influencing supine breathing.

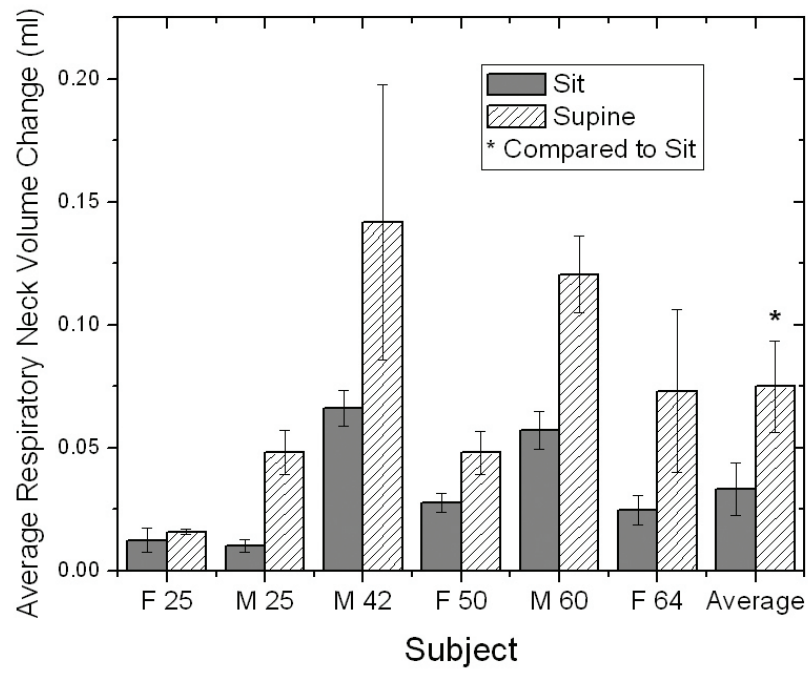

Figure 5. Average respiratory fluid shift while sitting and supine

The average respiratory fluid shift during four testing sessions while sitting and supine. Spectral analysis demonstrated a greater neck volume change during inhalation when supine in all subjects compared to sitting. Average respiratory neck volume change when supine was $0.08 \mathrm{ml} \pm 0.02$; 95\% CI [0.04, 0.12], significantly greater than during sitting $0.03 \mathrm{ml} \pm 0.01$; 95\% CI [0.01,0.05] $(p<$ $.01)$.

We noted significant increases in NV changes associated with breathing in both the seated and supine positions, with NV increasing with each inspiration. This was likely due to compression of the soft structures in the chest resulting in fluid being driven both rostrally and caudally. Moreover, the increase in NV during supine breathing was significantly greater (more than twice) that observed during seated breathing. Although the average NV change during supine breath- 
ing seems relatively small at $0.08 \pm 0.02 \mathrm{ml}$ with each breathe, the result can be clinically relevant. Neck fluid shifts may extravasate into the surrounding tissues building up extra-luminal pressure around the collapsible pharynx. According to Laplace's equation, resistance of an airway is inversely proportional to the fourth power of the change in radius of the airway indicating that small changes in the radius of the airway create large differences in terms of resistance thus lending to the propensity of an obstructive episode and its importance to nighttime breathing. Conversely, a small but significant improvement in airway resistance from an intervention may provide profound clinical improvements in individuals with SDB. Therefore, if fluid shifting into the neck when supine is associated with sleep disturbance, we suggest that this specific breathing component is the more likely factor.

There is evidence that RFS, as a result of daytime lower extremity fluid pooling, contributes to SDB such as obstructive sleep apnea hypopnea (OSAH). ${ }^{[9,10,13]}$ The cumulative effects of OSAH have been linked to many chronic cardiovascular conditions including, insulin resistance, congestive heart failure, myocardial ischemia, and even death. ${ }^{[18-22]}$ The results of the current study contribute to the characterization of the pattern of RFS through direct measurement of NV by utilizing the technique of APG, thus providing insight on additional possible methods to reduce RFS. Interventions to reduce fluid shifts towards the neck may provide effective therapies for OSAH. Further study of neck fluid shifts during respiration and its influence on sleep quality in persons with SDB is proposed.

While the APG measurements have yielded consistent results allowing us to more fully define fluid shifts in the upper airway, this study does have several limitations. First, the small, non-randomized sample of convenience limits the generalizability and inferences of this study. Additionally, our data indicated that 2 of the subjects contributed minimally to the effect of repositioning on NV. Redolfi et al. noted that change in leg fluid volume was related to daytime sitting and these two subjects may have been more active. ${ }^{[10]}$ Second, the small sample size may have introduced a Type II error resulting in non-significant results related to the differences between groups. We attempted to minimize variability by utilizing the same investigator (LB) to perform all testing, and precautions were taken to isolate the cuff from contact to eliminate external pressure against the cuff, but the coefficient of variation on our assessments was still close to $30 \%$. We believe that increased experience with this technology is likely to improve the reproducibility of these measurement techniques for assessing NV changes. Finally, while we calibrated our system to permit averaging of the NV change among our subjects, these calibration values do not reflect the absolute fluid volume changes in the neck. A detailed biomechanical model would allow us to convert our relative values to absolute fluid volume shifts.

\section{Conclusion}

In summary, APG was utilized to assess neck volume changes as a surrogate measure of the fluid shifts occurring within the neck during quiet sitting and in the supine position over a relatively moderately long time period (4560 minutes). Significant increases in NV were observed in both seated and supine positions. However, a significant increase in the dynamic respiratory component of $\mathrm{NV}$ was observed when supine, suggesting that respiratory activity may have the dominant influence on neck fluid and therefore on the soft structures in the neck, when supine. As described by Laplace's equation, the small volume changes during respiration that were recorded in this study, have the potential to impact airway resistance and thus nighttime breathing in a clinically significant manner. These results lead us to suggest that determination of the relationship between supine respiratory fluid shifts and neck volume adding to the understanding of RFS physiology thereby improving sleep assessment in adults.

\section{ACKNOWLEDGEMENTS}

The authors express our appreciation to all the participating volunteers and acknowledge the support of the Clinical Science and Engineering Research Center and the Decker School of Nursing at Binghamton University.

\section{REFERENCES}

[1] Rowell LB. Human Cardiovascular Control. New York: Oxford University Press, Inc.; 1993.

[2] Hagan RD, Diaz FJ, Horvath SM. Plasma Volume changes with movement to supine and standing positions. J Appl Physiol. 1978; 45(3): 414-7. PMID:701126.

[3] Padberg F. The physiology and hemodynamics of the normal venous circulation. In: Gloviczki P, Yao J, editors. Handbook of Venous
Disorders. 2nd ed. New York: Arnold Publishers; 2001. 25-35 p.

[4] Hildebrandt W, Gunga HC, Herrmann J, et al. Enhanced slow caudad fluid shifts in orthostatic intolerance after 24-h bed-rest. Eur J Appl Physiol Occup Physiol. 1994; 69: 61-70. PMID:7957158. http://dx.doi.org/10.1007/BF00867929

[5] Sjostrand T. Volume and distribution of blood and their significance in regulating the circulation. Am Physiol Soc Physiol Rev. 1953; 33: 202-28. 
[6] Tomaselli C, Kenney R, Frey M, et al. Cardiovascular dynamics during initial period of head-down tilt. Aviat Space Environ Med. 1987; 58: 3-8. PMID:3814029.

[7] Bradley TD, Floras JS. Sleep apnea and heart failure: Part I: obstructive sleep apnea. Circulation [Internet]. 2003 Apr 1 [cited 2013 May 26]; 107(12): 1671-8. Available from: http://www.ncbi.nlm.n ih.gov/pubmed/12668504

[8] Shepard JW, Pevernagie DA, Stanson AW, et al. Effects of changes in central venous pressure on upper airway size in patients with obstructive sleep apnea. Am J Respir Crit Care Med. 1996 Jan; 153(1): 250-4. PMID:8542124. http://dx.doi.org/10.1164/ajrccm. 153.1 .8542124

[9] Yumino D, Redolfi S, Ruttanaumpawan P, et al. Nocturnal rostral fluid shift: a unifying concept for the pathogenesis of obstructive and central sleep apnea in men with heart failure. Circulation [Internet]. 2010 Apr 13 [cited 2013 May 26]; 121(14): 1598-605. Available from: http://www.ncbi.nlm.nih.gov/pubmed/20351237

[10] Redolfi S, Yumino D, Ruttanaumpawan P, et al. Relationship between overnight rostral fluid shift and Obstructive Sleep Apnea in nonobese men. Am J Respir Crit Care Med [Internet]. 2009 Feb 1 [cited 2013 May 26]; 179(3): 241-6. Available from: http: //www.ncbi.nlm.nih.gov/pubmed/19011149

[11] Redolfi S, Arnulf I, Pottier M, et al. Effects of venous compression of the legs on overnight rostral fluid shift and obstructive sleep apnea. Respir Physiol Neurobiol [Internet]. Elsevier B.V.; 2011 Mar 15 [cited 2013 Nov 13]; 175(3): 390-3. Available from: http://www.ncbi.nlm.nih.gov/pubmed/21220055

[12] Su MC, Chiu KL, Ruttanaumpawan P, et al. Lower body positive pressure increases upper airway collapsibility in healthy subjects. Respir Physiol Neurobiol [Internet]. 2008 May 31 [cited 2013 Nov 7]; 161(3): 306-12. Available from: http://www.ncbi.nlm.nih .gov/pubmed/18439881

[13] Chiu KL, Ryan CM, Shiota S, et al. Fluid shift by lower body positive pressure increases pharyngeal resistance in healthy subjects. Am J Respir Crit Care Med [Internet]. 2006 Dec 15 [cited 2013 May 26]; 174(12): 1378-83. Available from: http://www.ncbi.nlm.nih.g ov/pubmed/16998093

[14] Nicolaides AN. Investigation of Chronic Venous Insufficiency: A Consensus Statement. Circulation. 2000; 102: e126-63.
PMID:11076834. http://dx.doi.org/10.1161/01.CIR.102. 20.e126

[15] Goddard A, Pierce CS, McLeod KJ. Reversal of lower limb edema by calf muscle pump stimulation. J Cardiopulm Rehabil Prev [Internet]. 2008; 28(3): 174-9. PMID:18496315. http://dx.doi.org /10.1097/01.HCR.0000320067.58599.ac

[16] Avasthey P, Wood E. Intrathoracic and venous pressure relationships during responses to changes in body position. J Appl Physiol. 1974; 37(2): 168-75.

[17] Atwood CW, Strollo PJ, Givelber R. Medical Therapy for Obstructive Sleep Apnea. In: Kryger M, Roth T, Dement W, editors. Principles and Practice of Sleep Medicine. 5th ed. St. Louis: Elsevier Saunders; 2011. 1219-32 p. http://dx . doi . org/10.1016/B978-1-4 160-6645-3.00106-7

[18] Polotsky VY, Jun J, Punjabi NM. Obstructive Sleep Apnea and Metabolic Dysfunction. In: Kryger M, editor. Principles and Practice of Sleep Medicine. 5th ed. Missouri: Elsevier Saunders; 2011. 13318 p. http://dx.doi.org/10.1016/B978-1-4160-6645-3.0 0114-6

[19] Punjabi NM, Ahmed MM, Polotsky VY, et al. Sleep-disordered breathing, glucose intolerance, and insulin resistance. Respir Physiol Neurobiol [Internet]. 2003 Jul [cited 2013 Oct 10]; 136(2-3): 167-78. Available from: http://linkinghub.elsevier.com/retrieve /pii/S156990480300079X

[20] Gottlieb DJ, Yenokyan G, Newman AB, et al. Prospective study of obstructive sleep apnea and incident coronary heart disease and heart failure: the sleep heart health study. Circulation [Internet]. $2010 \mathrm{Jul}$ 27 [cited 2013 May 21]; 122(4): 352-60. Available from: http: //www.pubmedcentral.nih.gov/articlerender.fcgi?art id=3117288\&tool=pmcentrez\&rendertype=abstract

[21] Sharma SK, Agrawal S, Damodaran D, et al. CPAP for the metabolic syndrome in patients with obstructive sleep apnea. N Engl J Med. 2011 Dec 15; 365(24): 2277-86. PMID:22168642. http://dx.doi .org/10.1056/NEJMoa1103944

[22] Verrier R, Mittleman M. Sleep-related Cardiac Risk. In: Kryger M, Roth T, Dement W, editors. Principles and Practice of Sleep Medicine. 5th ed. St. Louis: Elsevier Saunders; 2011. 1353-62 p. http: //dx.doi.org/10.1016/B978-1-4160-6645-3.00117-1 